Bol. Soc. Bot. México 49:3- 5.(1989)

\title{
La fisiología ecológica de plantas
}

En la explicación de las causas de la distribución y abundancia de los organismos vivos y de los fenómenos de interacción, los ecólogos tradicionalmente llegaban hasta el nivel de interpretación que les permitían las armas estadísticas a su disposición, pero no estaban preparados para conocer las causas profundas de lo observado y en muchas ocasiones eran incapaces de realizar experimentos en que en alguna medida se reflejara la realidad en la que estaban teniendo lugar los procesos vitales de sus objetos de estudio, en conexión con el problema analizado.

Abundan en la literatura ecológica, aún en la más escogida, los trabajos de pobre calidad en los que se nota una carencia profunda de conocimientos sobre las condiciones en las que se dan los procesos fisiológicos y la naturaleza de estos en las plantas estudiadas. Muchos experimentos describen condiciones experimentales en las que resulta evidente que se alteran, a veces radicalmente, las condiciones ambientales naturales, sin que los investigadores hayan sido capaces de apreciarlo.

Por otra parte, la fisiología tradicional contiene los conocimientos y las armas metodológicas para penetrar en los aspectos causales del comportamiento de las plantas para un número limitado de especies en condiciones controladas. Es por esto que la fisiología ecológica es una ciencia multidisciplinaria por excelencia, porque al llevar los conocimientos y la metodología de la fisiología vegetal al campo, se abren las puertas a una interpretación más profunda del funcionamiento de los sistemas vivos, y para ello se hace uso de conocimientos complementarios de otras disciplinas como la microclimatología, la edafología, la biofísica, la química e incluso de los aspectos más avanzados de la ingeniería y la informática. Esto, aunado a los avances en genética de poblaciones, está permitiendo el rompimiento de la frontera que limita a la ecología descriptiva tradicional.

En este número especial se presentan trabajos que se enfocan a algunos aspectos básicos de la ecofisiología de plantas. Temas que actualmente se desarrollan a gran velocidad y adquieren niveles crecientes de complejidad gracias a las contínuas mejoras que ocurren en la metodología empleada para la obtención de datos fisiológicos, físicos, y microclimáticos entre otros. El lector podrá apreciar no solo el nivel de conocimientos alcanzados sino también las óptimas posibilidades de aplicación que tiene el conocimiento generado en este campo, tanto a problemas de uso y manejo de recursos como en agronomía.

Con el fin de complementar esta breve introducción al presente número, se ofrece aquí una lista bibliográfica de libros que de una manera directa o indirecta cubren diversos tópicos de fisiología ecológica de plantas o intentan ser una síntesis al nivel de libros de texto. No se pretende que esta lista sea exaustiva, ya que el número de textos crece día con día y muchos de ellos abordan aspectos más específicos como la ecofisiología de la fotosíntesis, el metabolismo del nitrógeno, etcétera. 
Los autores que prepararon los artículos presentados en este número participaron en el Simposio de Ecofisiología que tuvo lugar durante el Décimo Congreso Mexicano de Botánica que se llevó a cabo en Guadalajara, Jalisco durante 1987.

Carlos Vazquez-Yanes. Centro de Ecología Universidad Nacional Autónoma de México, Apdo. Postal, 70-275, Delegación Coyoacán, 04510, México, D. F.

\section{LISTA DE UIBROS BASICOS POSTERIORES A 1975}

ALVIM DE T. y T. T. KOZLOWSKI. 1977. Ecophysiology of tropical crops. Academic Press, New York. BANNISTER, P. 1976. Introduction to physiological ecology. Blackwell Scientific Publications, Oxford.

BARBOUR, M. G., J. H. BURK y W. D. PITTS. 1980. Terrestrial plant ecology. Benjamin \& Cummings Publications, Menlo Park.

BARON, V. M. M. 1979. Organization in plants. Edward Arnold, London.

BOX, E. O. 1981. Macroclimate and plant form. Dr. W. Junk Publishers, The Hague.

CAMPBELL, G. S. 1977. An introduction to environmental biophysics. Springer Verlag. New York, Heidelberg.

COX, W. G. y M. D. ATKINS. 1979. Agricultural ecology. W. H. Freeman, San Francisco.

CRAWLEY, M. J. (Edr.). 1979. Plant ecology. Blackwell Scientific Publications, Oxford.

CHABOT, B. F. y H. A. MOONEY (Edrs.). 1985. Physiological plant ecology of North American plant communities. Chapman \& Hall, New York.

CHRISTIANSEN, M. M. y C. F. LEWIS (Edrs.). 1982. Breeding plants for less favourable environments. John Wiley \& Sons, New York.

DAUBENMIRE, R. F. 1979. Ecología vegetal: tratado de autoecología de plantas. Limusa Wiley, México D.F.

DIRZO, R. y J. SARUKHAN (Edrs.). 1984. Perspectives in plant population ecology. Sinauer Associates, Sunderland Mass.

ETHERINGTON, J. R. 1982. Environment and plant ecology . John Wiley \& Sons, London.

FENNER, M. 1986. Seed ecology. Chapman \& Hall, London.

FITTER, A. H. y R. K. M. HAY. 1981. Environmental physiology of plants. Academic Press, London.

GIVNISH, T. J. (Edr.). 1986. On the economy of plant form and function. Cambridge University Press, London.

GRIME, J. P. 1979. Plant strategies and vegetation processes. John Wiley \& Sons, London.

HARPER, J. L. 1977. Population biology of plants. Academic Press, London.

JOHNSON, C.B. (Edr.). 1981. Physiological processes limiting plant productivity. Butterworths, London. JONES, H. G. 1985. Plants and microclimate. Cambridge University Press, London.

KRAMER, P. J. y T. T. KOZLOWSKI. 1979. Physiology of woody plants. Academic Press, London.

LANGE, O. L., P. S. NOBEL, C. B. OSMOND y H. ZIEGLER (Edrs.). 1981. Plant physiological ecology.

En: Encyclopedia of plant physiology (New Series Vol. 12.) Springer Verlag, Berlin. 


\section{ECOFISIOLOGIA}

LARCHER, W. 1978. Physiological plant ecology. Springer Verlag, Berlin.

LEMEE, G. 1978. Precis d'ecologie vegetale. Masson et Cie. Editeurs, París.

LEVITT, J. 1980. Responses of plants to environmental stresses, vol. I: chilling, freezing and high temperature stresses, vol II water, radiation, salt and other stresses. Academic Press, New York.

LOVELESS, A. R. 1983. Principles of plant biology for the tropics. Longman, London.

MEDINA, E. 1977. Introducción a la ecofisiología vegetal. Programa de Desarrollo Científico y Tecnológico, Organización de Estados Americanos, Washington D.C.

MEDINA, E., H. A. MOONEY y C. VAZQUEZ-YANES. 1984. Physiological ecology of plants of the wet tropics. Dr. W. Junk Publishers, The Hague.

NOBEL, P. S. 1980. Biophysical plant physiology and ecology. W. H. Freeman, San Francisco.

NOBEL, P. S. 1988. Environmental biology of Agaves and Cacti. Cambridge University Press, N. Y.

OSMOND, C. B., O. BJÖRKMAN y D. J. ANDERSON. 1980. Physiological processes in plant ecology: toward a synthesis with Atriplex. Springer Verlag, Berlín.

PEARCY, R. W., J. EHLERINGER, H. A. MOONEY y P. W. RUNDEL. 1989. Plant physiological ecology. Field methods and Instrumentation. Chapman and Hall, London.

ROSS, J. 1982. The radiation regime and architecture of plants. Dr. W. Junk Publishers, The Hague.

SARMIENTO, G. 1984. The ecology of neotropical savannas. Harvard University Press, Cambridge Mass. SMITH, H. (Edr.). 1976. Light and plant development. Butterworths, London.

SOLBRIG, O. T., S. JAIN, G. B. JOHNSON y P. H. RAVEN (Edrs.) 1979. Topics in plant population biology. Columbia University Press, New York.

TEAS, H. J. (Edr.). 1984. Physiology and management of mangroves. Dr. W. Junk Publishers, The Hague.

TOWSEND, C. R. y P. R. CALOW (Edrs.). 1981. Physiological ecology, an evolutionary approach to resource use. Sinauer Associates, Sunderland Mass.

TURNER, N. C. y P. J. KRAMER. 1981. Adaptation of plants to water and high temperature stress. John Wiley \& Sons, London.

VAZQUEZ-YANES, C. 1987. Cómo viven las plantas. Fondo de Cultura Económica, México D.F.

VICKERY, M. L. 1984. Ecology of tropical plants. John Wiley \& Sons, London.

WALTER, H. y S. W. BRECKLE. 1985. Ecological systems of the geobiosphere. Springer Verlag, Berlin. 